\title{
Modified Cut-Off Value of the Urine Protein-To-Creatinine Ratio Is Helpful for Identifying Patients at High Risk for Chronic Kidney Disease: Validation of the Revised Japanese Guideline
}

\author{
Hiroyuki Yamamoto, ${ }^{1,2}$ Kyoko Yamamoto, ${ }^{1,3,4}$ Katsumi Yoshida, ${ }^{5}$ \\ Chiyohiko Shindoh, ${ }^{3}$ Kyoko Takeda, ${ }^{6}$ Masami Monden, ${ }^{7}$ Hiroko Izumo, ${ }^{7}$ \\ Hiroyuki Niinuma, ${ }^{8}$ Yutaro Nishi, ${ }^{8}$ Koichiro Niwa ${ }^{8}$ and Yasuhiro Komatsu ${ }^{1}$ \\ ${ }^{1}$ Division of Nephrology, Department of Internal Medicine, St. Luke's International Hospital, Tokyo, Japan \\ ${ }^{2}$ Oiwa Nephrology and Dialysis Clinic, Tokyo, Japan \\ ${ }^{3}$ Department of Laboratory Medicine and Clinical Science, Tohoku University Graduate School of Medicine, \\ Sendai, Miyagi, Japan \\ ${ }^{4}$ Department of Thoracic Medical Oncology, Cancer Institute Hospital of Japanese Foundation for Cancer \\ Research, Tokyo, Japan \\ ${ }^{5}$ Healthy Medical Center, Tohoku Kosai Hospital, Sendai, Miyagi, Japan \\ ${ }^{6}$ Clinical Laboratory Department, St. Luke's International Hospital, Tokyo, Japan \\ ${ }^{7}$ Division of Endocrinology and Metabolism, Department of Internal Medicine, St. Luke's International Hospital, \\ Tokyo, Japan \\ ${ }^{8}$ Department of Cardiology, Cardiovascular Center, St. Luke's International Hospital, Tokyo, Japan
}

Chronic kidney disease (CKD) is a global public health issue, and strategies for its early detection and intervention are imperative. The latest Japanese CKD guideline recommends that patients without diabetes should be classified using the urine protein-to-creatinine ratio (PCR) instead of the urine albumin-tocreatinine ratio (ACR); however, no validation studies are available. This study aimed to validate the PCR-based CKD risk classification compared with the ACR-based classification and to explore more accurate classification methods. We analyzed two previously reported datasets that included diabetic and/ or cardiovascular patients who were classified into early CKD stages. In total, 860 patients (131 diabetic patients and 729 cardiovascular patients, including 193 diabetic patients) were enrolled. We assessed the CKD risk classification of each patient according to the estimated glomerular filtration rate and the ACR-based or PCR-based classification. The use of the cut-off value recommended in the current guideline (PCR $0.15 \mathrm{~g} / \mathrm{g}$ creatinine) resulted in risk misclassification rates of $26.0 \%$ and $16.6 \%$ for the two datasets. The misclassification was primarily caused by underestimation. Moderate to substantial agreement between each classification was achieved: Cohen's kappa, 0.56 (95\% confidence interval, 0.45 $0.69)$ and $0.72(0.67-0.76)$ in each dataset, respectively. To improve the accuracy, we tested various candidate PCR cut-off values, showing that a PCR cut-off value of $0.08-0.10 \mathrm{~g} / \mathrm{g}$ creatinine resulted in improvement in the misclassification rates and kappa values. Modification of the PCR cut-off value would improve its efficacy to identify high-risk populations who will benefit from early intervention.

Keywords: chronic kidney disease; Cohen's kappa statistic; urine albumin-to-creatinine ratio; urine protein-tocreatinine ratio; validation

Tohoku J. Exp. Med., 2015 November, 237 (3), 201-207. C 2015 Tohoku University Medical Press

\section{Introduction}

The publication of the chronic kidney disease (CKD) guideline by the National Kidney Foundation in 2002 resulted in a paradigm shift in both public health and nephrology (National Kidney Foundation 2002). CKD has been recognized as a public health issue that poses risks for cardiovascular disease and end-stage kidney disease (Schieppati and Remuzzi 2005; Levey et al. 2007). The World Health Organization (WHO) defined CKD as one of

Received January 13, 2015; revised and accepted September 29, 2015. Published online October 24, 2015; doi: 10.1620/tjem.237.201. Correspondence: Hiroyuki Yamamoto, M.D., Ph.D., MPH, Division of Nephrology, Department of Internal Medicine, St. Luke's

International Hospital, 9-1 Akashi-cho, Chuo-ku, Tokyo 104-8560, Japan.

e-mail: yama-h@umin.ac.jp

Yasuhiro Komatsu, M.D., Ph.D., MPH, Division of Nephrology, Department of Internal Medicine, St. Luke's International Hospital,

9-1 Akashi-cho, Chuo-ku, Tokyo 104-8560, Japan.

e-mail: komayasu@luke.ac.jp 
the non-communicable diseases that require public attention (World Health Organization 2011). Emerging evidence has led to a revision of the definition of CKD to further identify high-risk populations who require early intervention to prevent cardiovascular disease and the progression of kidney disease (Chronic Kidney Disease Prognosis Consortium et al. 2010; Gansevoort et al. 2011; Levey et al. 2011). The original definition included only the estimated glomerular filtration rate (eGFR), whereas the most recent definition proposed by Kidney Disease: Improving Global Outcomes (KDIGO) Clinical Practice Guideline adopted the combination of the cause of disease, the glomerular filtration rate (GFR) and albuminuria (KDIGO CKD Work Group 2013).

The Japanese Society of Nephrology also revised the Japanese CKD guideline in 2013, recommending that the CKD stage be classified according to GFR and albuminuria categories (Japanese Society of Nephrology 2014). Because the Japanese National Health Insurance program reimburses the measurement of urine albumin-to-creatinine ratio (ACR) only for diabetes patients, the Japanese guideline proposes the use of the urine protein-to-creatinine ratio (PCR) instead of the ACR. This guideline assumes that a PCR of $0.15 \mathrm{~g} / \mathrm{g}$ creatinine is equivalent to an ACR of 30 $\mathrm{mg} / \mathrm{g}$ creatinine and that this value should be used as the cut-off for differentiating albuminuria categories. However, this assumption is based on incomplete evidence, and no validation studies are available. The latest studies of the relationship between ACR and PCR have indicated that an ACR of $30 \mathrm{mg} / \mathrm{g}$ creatinine is equal to a PCR of slightly lower than $0.15 \mathrm{~g} / \mathrm{g}$ creatinine (Yamamoto et al. 2011, 2014; Fisher et al. 2013). These results challenge the validity of the PCR-based classification method recommended by the Japanese CKD guideline.

This study aimed to (i) describe and validate the PCRbased CKD risk classification method recommended by the current Japanese CKD guideline using the ACR-based classification method as the gold standard and (ii) to explore more appropriate cut-off values using secondary datasets.

\section{Materials and Methods}

Data source

This study was based on a secondary analysis of our two previous outpatient studies, with 150 diabetic patients included in the first study (2011) (hereafter, Study 1) (Yamamoto et al. 2011) and 784 cardiovascular patients included in the second study (2014) (hereafter, Study 2) (Yamamoto et al. 2014). The methods and primary results were reported in detail in each article. In this study, the age, sex, serum creatinine concentration, urine dipstick test results, ACR, and PCR of the patients were determined from the previous datasets. This study was approved by the Research Ethics Committee of St. Luke's International Hospital in Tokyo.

\section{Classification of CKD stage and severity}

The Japanese Society of Nephrology and KDIGO CKD guidelines recommend that the CKD stage of each patient should be classified by a 6-by-3 matrix according to the GFR (G1, G2, G3a, G3b, G4, and G5) and albuminuria categories (A1, A2, and A3) (KDIGO CKD
Work Group 2013; Japanese Society of Nephrology 2014). These guidelines advocate the use of risk classification as generated by a 'heat map' of CKD stage matrices, which highlights future risk and the required interventions for each patient in a clinical setting (Levin and Stevens 2014). The CKD risk classification is demonstrated by the color intensity (see Fig. 1), which represents the prognosis and risk of CKD progression.

To determine the CKD risk classification of each patient, the eGFR and ACR of each patient were assessed. The eGFR was calculated using the following formula for the Japanese population: eGFR $\left(\mathrm{mL} / \mathrm{min} / 1.73 \mathrm{~m}^{2}\right)=194 \times$ serum creatinine ${ }^{-1.094}(\mathrm{mg} / \mathrm{dL}) \times$ age $^{-0.287}$ (years) $\times 0.739$ (if female) (Matsuo et al. 2009). According to the eGFR and ACR, each patient was classified into an independent CKD risk classification matrix (using the ACR-based classification method as the gold standard). Patients classified as G1-G3b in the GFR category and as A1 and A2 in the albuminuria category were enrolled in this study because our interest was the utility of the PCR-based method in normal to relatively early-stage CKD patients. Thus, 19 patients from Study 1 and 55 patients from Study 2 were excluded. For comparison, several candidate cut-off PCR values ( 0.05 to $0.15 \mathrm{~g} /$ $\mathrm{g}$ creatinine) were utilized as alternatives to the ACR-based method to discriminate between the $\mathrm{A} 1$ and $\mathrm{A} 2$ albuminuria categories.

\section{Evaluation of agreement}

First, the misclassification rate for the PCR-based CKD risk classification method was calculated and compared with that of the ACR-based method. Cohen's kappa statistics were calculated to evaluate the agreement between each classification method (Landis and Koch 1977; Kundel and Polansky 2003). A kappa statistic is a summary measurement of agreement that considers the measurement of a chance agreement (Sim and Wright 2005; Viera and Garrett 2005). The $95 \%$ confidence intervals of the kappa statistics were estimated by the Stata command "kapci" using bootstrap methods with 1,000 replications (Reichenheim 2004). Interpretation of the kappa statistics was performed as in previous studies (Kundel and Polansky 2003). A kappa value $\leq 0.20$ was considered poor, 0.21-0.40 was considered slight, 0.41-0.60 was considered moderate, 0.61-0.80 was considered substantial, and 0.81-1.00 was considered almost perfect.

\section{Data analyses}

The numerical data are expressed as the mean and standard deviation for normal distributions and as the median (interquartile range) for non-normal distributions. The categorical data are expressed as percentages. We analyzed the two datasets separately because the results obtained from a combined dataset would have been greatly influenced by a large sample size. Statistical analyses were conducted using Stata 13 software (Stata Corp, College Station, TX, USA).

\section{Results}

\section{Patient characteristics and CKD risk classification}

A total of 860 patients (131 patients from Study 1 and 729 patients from Study 2) were enrolled in this study (Table 1). All of the patients in Study 1 and $26.5 \%$ of those in Study 2 had diabetes mellitus (DM). The mean eGFR in both studies was approximately $70 \mathrm{~mL} / \mathrm{min} / 1.73 \mathrm{~m}^{2}$. The prevalence of albuminuria in the patients from Study 1 and Study 2 was $43.5 \%$ and $33.1 \%$, respectively. The CKD risk 
Table 1. Baseline patient characteristics.

\begin{tabular}{|c|c|c|}
\hline Characteristics & $\begin{array}{c}\text { Study } 1 \\
(\mathrm{~N}=131)\end{array}$ & $\begin{array}{c}\text { Study } 2 \\
(\mathrm{~N}=729)\end{array}$ \\
\hline Age (years) & $\begin{array}{c}64.4 \pm 12.5 \\
(\text { range }=26-87)\end{array}$ & $\begin{array}{c}68.5 \pm 11.8 \\
(\text { range }=29-98)\end{array}$ \\
\hline Male (\%) & $60(45.8)$ & $464(63.7)$ \\
\hline Albuminuria (\%) & $57(43.5)$ & $241(33.1)$ \\
\hline Diabetes mellitus (\%) & $131(100)$ & $193(26.5)$ \\
\hline \multicolumn{3}{|l|}{ Laboratory test } \\
\hline Serum creatinine $(\mathrm{mg} / \mathrm{dL})$ & 0.74 (IQR 0.26) & 0.78 (IQR 0.29) \\
\hline eGFR $\left(\mathrm{mL} / \mathrm{min} / 1.73 \mathrm{~m}^{2}\right)$ & $73.4 \pm 18.4$ & $71.3 \pm 18.9$ \\
\hline PCR (g/g creatinine) & 0.073 (IQR 0.077) & 0.069 (IQR 0.076) \\
\hline ACR (mg/g creatinine) & 23.8 (IQR 51.0) & 17.5 (IQR 33.1) \\
\hline \multicolumn{3}{|l|}{ Proteinuria by dipstick test } \\
\hline$-(\%)$ & $87(66.4)$ & 517 (70.9) \\
\hline$\pm(\%)$ & $28(21.4)$ & $141(19.3)$ \\
\hline $1+(\%)$ & $14(10.7)$ & $71(9.7)$ \\
\hline $2+(\%)$ & $2(1.5)$ & $0(0)$ \\
\hline
\end{tabular}

The data are presented as the mean \pm standard deviation or as the median (interquartile range) as appropriate.

eGFR, estimated glomerular filtration rate; PCR, urine protein-to-creatinine ratio; ACR, urine albuminto-creatinine ratio; IQR, interquartile range.

A

\begin{tabular}{|c|c|c|c|c|c|}
\hline \multirow[t]{2}{*}{ Study } & \multicolumn{4}{|c|}{ PCR $0.15 \mathrm{~g} / \mathrm{gCr}$} & Total \\
\hline & 56 & 1 & 0 & 0 & 57 \\
\hline \multirow{3}{*}{$\begin{array}{c}\text { ACR } \\
30 \mathrm{mg} \\
/ \mathrm{gCr}\end{array}$} & 24 & 32 & 0 & 0 & 56 \\
\hline & 0 & 8 & 7 & 0 & 15 \\
\hline & 0 & 0 & 1 & 2 & 3 \\
\hline Total & 80 & 41 & 8 & 2 & 131 \\
\hline
\end{tabular}

B

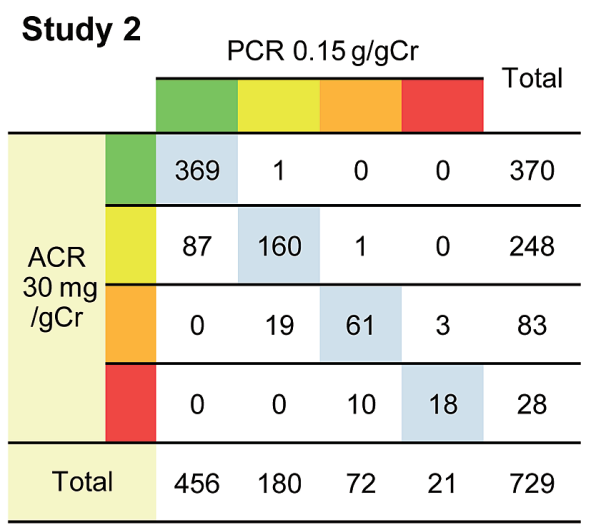

Fig. 1. Agreement between the ACR- and PCR-based CKD risk classification methods.

Each column indicates the CKD risk classification determined using the ACR-based classification method, as demonstrated by the color intensity (green, low risk; yellow, moderately increased risk; orange, high risk; red, very high risk). Each row indicates the CKD risk classification using the PCR-based cut-off $(0.15 \mathrm{~g} / \mathrm{g}$ creatinine). The concordant pairs are highlighted with a blue background. Each cell contains the number of patients classified in Study 1 (A) and Study 2 (B).

CKD, chronic kidney disease; ACR, urine albumin-to-creatinine ratio; PCR, urine protein-to-creatinine ratio.

classifications of the patients as classified by the eGFR and ACR or PCR (cut-off value of $0.15 \mathrm{~g} / \mathrm{g}$ creatinine) are presented in Fig. 1.

\section{Misclassification rates of $C K D$ risk classification methods}

The use of a PCR cut-off of $0.15 \mathrm{~g} / \mathrm{g}$ creatinine resulted in misclassification rates of $26.0 \%$ and $16.6 \%$ of the patients in Study 1 and Study 2, respectively (Table 2). The observed misclassification was primarily (> 95\%) caused by the underestimation of the CKD risk classifica- tion (Fig. 1). Alternatively, the use of a PCR range of 0.08$0.10 \mathrm{~g} / \mathrm{g}$ creatinine as the cut-off value improved the misclassification rates. The use of a PCR cut-off of $0.09 \mathrm{~g} / \mathrm{g}$ creatinine resulted in a decrease in the misclassification rate to approximately $10 \%$ of the patients, with the equal occurrence of underestimation and overestimation.

\section{Agreement of CKD risk classification}

Fig. 2 presents the relationship between the PCRbased CKD risk classification method and the kappa statis- 
Table 2. Relationship between the cut-off value and misclassification rate of CKD risk classification.

\begin{tabular}{ccc}
\hline & \multicolumn{2}{c}{ Misclassification rate (\%) } \\
\cline { 2 - 3 } (equal to an ACR of $30 \mathrm{mg} / \mathrm{g} \mathrm{Cr})$ & $\begin{array}{c}\text { Study } 1 \\
\mathrm{~N}=131)\end{array}$ & $\begin{array}{c}\text { Study } 2 \\
\mathrm{~N}=729)\end{array}$ \\
\hline PCR (g/g Cr) & & 35.0 \\
0.05 & 33.6 & 23.2 \\
0.06 & 23.7 & 17.6 \\
0.07 & 14.5 & 12.4 \\
0.08 & 12.2 & 10.8 \\
0.09 & 9.9 & 10.2 \\
0.10 & 14.5 & 10.3 \\
0.11 & 21.4 & 11.4 \\
0.12 & 21.4 & 13.2 \\
0.13 & 23.7 & 15.1 \\
0.14 & 24.4 & 16.6 \\
0.15 & 26.0 & \\
\hline
\end{tabular}

$\mathrm{ACR}$, urine albumin-to-creatinine ratio; $\mathrm{Cr}$, creatinine; $\mathrm{PCR}$, urine protein-to-creatinine ratio.

tics with $95 \%$ confidence intervals in the two studies. The use of a PCR cut-off of 0.08-0.09 g/g creatinine for Study 1 and $0.08-0.12 \mathrm{~g} / \mathrm{g}$ creatinine for Study 2 resulted in kappa values that were in the "almost perfect" range. The use of PCR cut-off values of $0.15 \mathrm{~g} / \mathrm{g}$ creatinine for both studies resulted in kappa values that reached 0.56 (95\% CI, 0.45 0.69; "moderate" range) for Study 1 and 0.72 (95\% CI, 0.67-0.76; "substantial" range) for Study 2. These kappa values were significantly lower than those obtained with a PCR cut-off range of 0.08-0.09 g/g creatinine in Study 1 and $0.08-0.12 \mathrm{~g} / \mathrm{g}$ in Study 2. These results are almost identical to those of a subgroup analysis of non-DM and DM patients in Study 2 (Table 3).

\section{Discussion}

$\mathrm{CKD}$ is one of the non-communicable diseases that the WHO identified as requiring a public health approach. Classification using a combination of eGFR and the degree of albuminuria or proteinuria is predictive of future cardiovascular and kidney disease outcomes. High-risk populations can benefit from early intervention, such as diet modification and blood pressure control, to retard progression of the disease. Previous studies (KDIGO CKD Work Group 2013) exploring the method used to detect high-risk populations led to a revision of the definition and classification of CKD.

In this study, we validated the PCR-based classification method recommended by the Japanese CKD guideline and found a "moderate" to "substantial" range of agreement with the gold standard ACR-based method. We demonstrated that the use of PCR cut-off ranges of 0.08-0.09 and 0.08-0.12 g/g creatinine for the datasets of Study 1 and Study 2, respectively, to differentiate between the A1 and A2 stages significantly improved the agreement. The results of the two datasets were almost identical, supporting the validity and strength of our results. To the best of our knowledge, this study is the first to validate the PCR-based CKD classification method. Our results are important because of the paucity of reports on the simultaneous measurements of PCR and ACR in the literature.

The current guideline states that CKD should be classified based on the cause as well as the GFR and albuminuria categories. Using nationwide Japanese "specific health checkup" data, a recent study reported that the GFR category has a limited ability to independently predict cardiovascular events in CKD (the area under the curve value reached approximately 0.6), highlighting the importance of the albuminuria category (Terawaki et al. 2014). However, other studies have examined the relationship between the ACR and PCR (Atkins et al. 2003; Methven et al. 2010; Smith et al. 2012). Our group previously reported that the PCR is highly correlated with the ACR and that an ACR of $30 \mathrm{mg} / \mathrm{g}$ creatinine is equivalent to a PCR of approximately 0.08-0.09 g/g creatinine, based on the analyzed datasets (Yamamoto et al. 2011, 2014). Fisher et al. (2013) reported that the ACR and PCR are relatively similar and that an ACR of $30 \mathrm{mg} / \mathrm{g}$ creatinine is equivalent to a PCR of approximately $0.08-0.11 \mathrm{~g} / \mathrm{g}$ creatinine based on regression and Lowess smooth analyses in a recent large CKD cohort study. These results imply that the PCR cut-off is not precisely equal to $0.15 \mathrm{~g} / \mathrm{g}$ creatinine and that a lower PCR is more suitable than previously considered.

Our results demonstrated that the agreement between the ACR-based and PCR-based methods $(0.15 \mathrm{~g} / \mathrm{g}$ creatinine) is in the "moderate" to "substantial" range, with a 15.3 to $26.0 \%$ misclassification rate for the PCR-based method. This method exhibits a remarkably low prevalence of overestimation of the CKD stage and risk classification. The current PCR-based method is favorable in circumstances in which the harm of overestimation or overdiagno- 
A. Study 1

\begin{tabular}{lccc}
\multicolumn{2}{c}{ cut-off value } & kappa & $95 \% \mathrm{Cl}$ \\
& $\mathbf{0 . 0 5}$ & 0.47 & $0.35-0.61$ \\
& $\mathbf{0 . 0 6}$ & 0.63 & $0.50-0.73$ \\
& $\mathbf{0 . 0 7}$ & 0.77 & $0.66-0.86$ \\
& $\mathbf{0 . 0 8}$ & 0.80 & $0.70-0.89$ \\
PCR & $\mathbf{0 . 0 9}$ & 0.84 & $0.75-0.92$ \\
(g/g Cr) & $\mathbf{0 . 1}$ & 0.77 & $0.66-0.86$ \\
& $\mathbf{0 . 1 1}$ & 0.65 & $0.53-0.76$ \\
& $\mathbf{0 . 1 2}$ & 0.65 & $0.54-0.76$ \\
& $\mathbf{0 . 1 3}$ & 0.61 & $0.48-0.73$ \\
& $\mathbf{0 . 1 4}$ & 0.59 & $0.48-0.71$ \\
& $\mathbf{0 . 1 5}$ & 0.56 & $0.45-0.69$
\end{tabular}

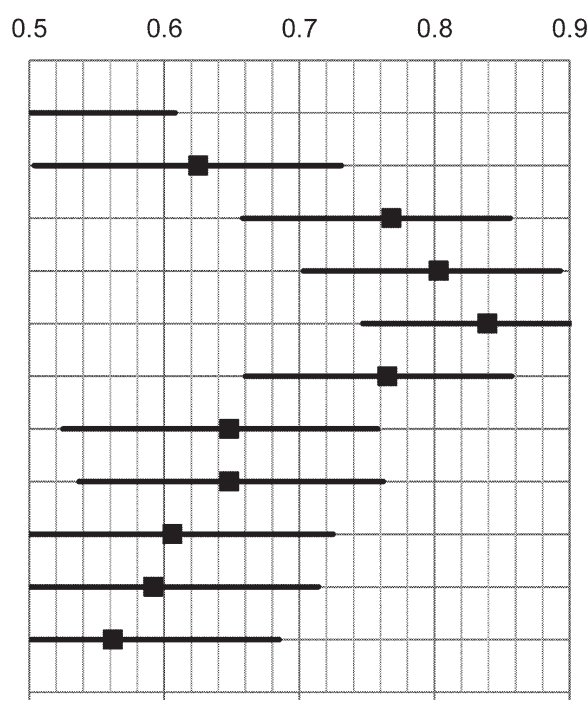

\section{B. Study 2}

\begin{tabular}{lccc}
\multicolumn{2}{c}{ cut-off value } & kappa & $95 \% \mathrm{Cl}$ \\
& $\mathbf{0 . 0 5}$ & 0.48 & $0.43-0.53$ \\
& $\mathbf{0 . 0 6}$ & 0.64 & $0.60-0.69$ \\
& $\mathbf{0 . 0 7}$ & 0.73 & $0.69-0.77$ \\
& $\mathbf{0 . 0 8}$ & 0.80 & $0.77-0.84$ \\
PCR & $\mathbf{0 . 0 9}$ & 0.83 & $0.79-0.86$ \\
(g/g Cr) & $\mathbf{0 . 1}$ & 0.83 & $0.80-0.87$ \\
& $\mathbf{0 . 1 1}$ & 0.83 & $0.79-0.86$ \\
& $\mathbf{0 . 1 2}$ & 0.81 & $0.77-0.85$ \\
& $\mathbf{0 . 1 3}$ & 0.78 & $0.73-0.82$ \\
& $\mathbf{0 . 1 4}$ & 0.74 & $0.70-0.78$ \\
& $\mathbf{0 . 1 5}$ & 0.72 & $0.67-0.76$
\end{tabular}

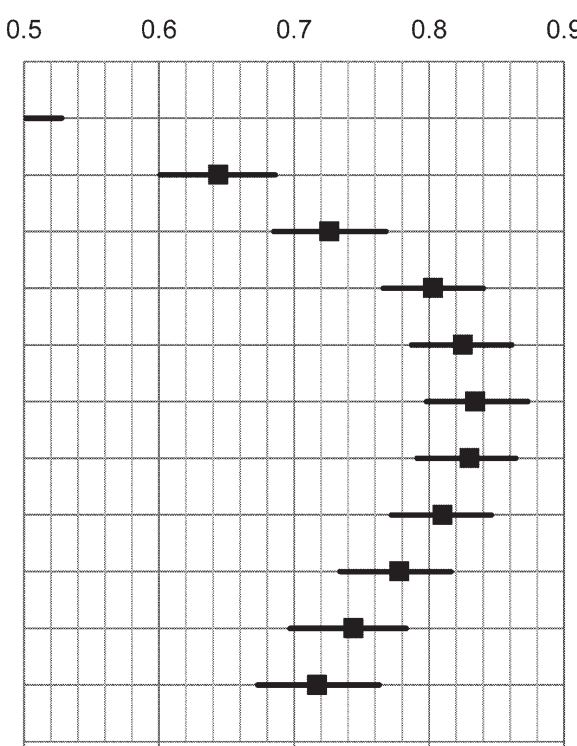

Fig. 2. Kappa statistics for the CKD risk classification according to each cut-off value.

The point estimates of the kappa statistics and the 95\% CI according to each cut-off value in Study 1 (A) and Study 2 (B) are demonstrated.

CI, confidence interval; PCR, urine protein-to-creatinine ratio.

sis outweighs the risk of underestimation. Overdiagnosis can lead to further medical workups, which can result in psychological and/or financial burdens (Moynihan et al. 2012). Clinicians should acknowledge the benefits and limitations of the current Japanese CKD guideline classification method when applying it in daily practice.

Another important implication of this study is that more efficient classification method would be applicable for both non-DM and DM patients and could be compared with the current PCR-based method. Misclassification of the CKD risk can lead to either undertreatment or overtreatment. (Levey et al. 2011; Kidney Disease: Improving Global Outcomes (KDIGO) CKD Work Group 2013). The appropriate alternative cut-off values mentioned above would improve the accuracy of the prediction of future risk for each patient and the comparability among international studies. Thus, future studies of the relationship between the ACR and PCR are required.

This study has the following limitations. First, it was conducted using secondary datasets in a single hospital outpatient setting. In addition, the study population was restricted to normal to relatively early-stage CKD. Second, this study was based on single measurement of the eGFR, ACR, and PCR. Third, the number of patients enrolled in Study 1 was small; therefore, the results of this study tended to be underpowered.

In conclusion, we have demonstrated that the current PCR-based classification method ( $0.15 \mathrm{~g} / \mathrm{g}$ creatinine) is 
Table 3. Relationship between the cut-off value and misclassification rate and Kappa statistics of CKD risk classification, including a diabetes mellitus subgroup from Study 2.

\begin{tabular}{|c|c|c|c|c|}
\hline \multirow{2}{*}{$\begin{array}{l}\text { Cut-off value } \\
\text { (equal to an ACR of } \\
30 \mathrm{mg} / \mathrm{g} \mathrm{Cr} \text { ) }\end{array}$} & \multicolumn{2}{|c|}{$\begin{array}{l}\text { Non-DM } \\
(\mathrm{N}=536)\end{array}$} & \multicolumn{2}{|c|}{$\begin{array}{c}\mathrm{DM} \\
(\mathrm{N}=193)\end{array}$} \\
\hline & $\begin{array}{c}\text { Misclassification rate } \\
(\%)\end{array}$ & $\begin{array}{l}\text { Kappa statistics } \\
(95 \% \mathrm{CI})\end{array}$ & $\begin{array}{c}\text { Misclassification rate } \\
(\%)\end{array}$ & $\begin{array}{c}\text { Kappa statistics } \\
(95 \% \mathrm{CI})\end{array}$ \\
\hline \multicolumn{5}{|l|}{ PCR (g/g Cr) } \\
\hline 0.05 & 35.8 & $0.46(0.41-0.52)$ & 32.6 & $0.51(0.42-0.61)$ \\
\hline 0.06 & 24.1 & $0.62(0.57-0.68)$ & 20.7 & $0.69(0.59-0.77)$ \\
\hline 0.07 & 17.9 & $0.71(0.66-0.76)$ & 16.6 & $0.75(0.67-0.82)$ \\
\hline 0.08 & 12.3 & $0.80(0.75-0.84)$ & 12.4 & $0.81(0.75-0.88)$ \\
\hline 0.09 & 10.5 & $0.82(0.78-0.87)$ & 11.9 & $0.82(0.75-0.90)$ \\
\hline 0.10 & 9.5 & $0.84(0.79-0.88)$ & 11.9 & $0.82(0.75-0.88)$ \\
\hline 0.11 & 9.5 & $0.84(0.79-0.87)$ & 12.4 & $0.81(0.73-0.87)$ \\
\hline 0.12 & 10.8 & $0.81(0.76-0.86)$ & 13.0 & $0.80(0.72-0.87)$ \\
\hline 0.13 & 12.9 & $0.77(0.72-0.82)$ & 14.0 & $0.79(0.71-0.86)$ \\
\hline 0.14 & 14.2 & $0.75(0.69-0.80)$ & 17.6 & $0.73(0.65-0.81)$ \\
\hline 0.15 & 15.3 & $0.72(0.67-0.77)$ & 20.2 & $0.69(0.60-0.78)$ \\
\hline
\end{tabular}

DM, diabetes mellitus; CI, confidence interval; ACR, urine albumin-to-creatinine ratio; PCR, urine protein-to-creatinine ratio.

highly specific, providing a certain level of validity; however, modification of the cut-off value (PCR values of approximately $0.08-0.10 \mathrm{~g} / \mathrm{g}$ creatinine) would further improve its efficacy and identify high-risk populations who will benefit from early intervention to prevent cardiovascular disease and progression to end-stage kidney disease.

\section{Acknowledgments}

This work was presented in part as an oral presentation at the 56th Annual Meeting of the Japanese Society of Nephrology, May 10-12, 2013, Tokyo, Japan, and was accepted in part as a poster presentation at the American Society of Nephrology Kidney Week 2015, November 3-8, 2015, San Diego, CA. This study was partially supported by a grant from St. Luke's Life Science Institute.

\section{Conflict of Interest}

The authors declare no conflict of interest.

\section{References}

Atkins, R.C., Briganti, E.M., Zimmet, P.Z. \& Chadban, S.J. (2003) Association between albuminuria and proteinuria in the general population: the AusDiab Study. Nephrol. Dial. Transplant., 18, 2170-2174.

Chronic Kidney Disease Prognosis Consortium; Matsushita, K., van der Velde, M., Astor, B.C., Woodward, M., Levey, A.S., de Jong, P.E., Coresh, J. \& Gansevoort, R.T. (2010) Association of estimated glomerular filtration rate and albuminuria with all-cause and cardiovascular mortality in general population cohorts: a collaborative meta-analysis. Lancet, $\mathbf{3 7 5}$, 2073-2081.

Fisher, H., Hsu, C.Y., Vittinghoff, E., Lin, F. \& Bansal, N. (2013) Comparison of associations of urine protein-creatinine ratio versus albumin-creatinine ratio with complications of CKD: a cross-sectional analysis. Am. J. Kidney Dis., 62, 1102-1108.

Gansevoort, R.T., Matsushita, K., van der Velde, M., Astor, B.C., Woodward, M., Levey, A.S., de Jong, P.E. \& Coresh, J.; Chronic Kidney Disease Prognosis Consortium (2011) Lower estimated GFR and higher albuminuria are associated with adverse kidney outcomes. A collaborative meta-analysis of general and high-risk population cohorts. Kidney Int., 80, 93-104.

Japanese Society of Nephrology (2014) Evidence-based Clinical Practice Guideline for CKD 2013. Clin. Exp. Nephrol., 18, 346-423.

Kidney Disease: Improving Global Outcomes (KDIGO) CKD Work Group (2013) KDIGO 2012 Clinical Practice Guideline for the Evaluation and Management of Chronic Kidney Disease. Kidney Int. Suppl., 3, 1-150.

Kundel, H.L. \& Polansky, M. (2003) Measurement of observer agreement. Radiology, 228, 303-308.

Landis, J.R. \& Koch, G.G. (1977) The measurement of observer agreement for categorical data. Biometrics, 33, 159-174.

Levey, A.S., Atkins, R., Coresh, J., Cohen, E.P., Collins, A.J., Eckardt, K.U., Nahas, M.E., Jaber, B.L., Jadoul, M., Levin, A., Powe, N.R., Rossert, J., Wheeler, D.C., Lameire, N. \& Eknoyan, G. (2007) Chronic kidney disease as a global public health problem: approaches and initiatives - a position statement from Kidney Disease Improving Global Outcomes. Kidney Int., 72, 247-259.

Levey, A.S., de Jong, P.E., Coresh, J., El Nahas, M., Astor, B.C., Matsushita, K., Gansevoort, R.T., Kasiske, B.L. \& Eckardt, K.U. (2011) The definition, classification, and prognosis of chronic kidney disease: a KDIGO Controversies Conference report. Kidney Int., 80, 17-28.

Levin, A. \& Stevens, P.E. (2014) Summary of KDIGO 2012 CKD Guideline: behind the scenes, need for guidance, and a framework for moving forward. Kidney Int., 85, 49-61.

Matsuo, S., Imai, E., Horio, M., Yasuda, Y., Tomita, K., Nitta, K., Yamagata, K., Tomino, Y., Yokoyama, H. \& Hishida, A.; Collaborators developing the Japanese equation for estimated GFR (2009) Revised equations for estimated GFR from serum creatinine in Japan. Am. J. Kidney Dis., 53, 982-992.

Methven, S., MacGregor, M.S., Traynor, J.P., O'Reilly, D.S. \& Deighan, C.J. (2010) Assessing proteinuria in chronic kidney disease: protein-creatinine ratio versus albumin-creatinine ratio. Nephrol. Dial. Transplant., 25, 2991-2996.

Moynihan, R., Doust, J. \& Henry, D. (2012) Preventing overdiagnosis: how to stop harming the healthy. BMJ, 344, e3502.

National Kidney Foundation (2002) K/DOQI clinical practice 
guidelines for chronic kidney disease: evaluation, classification, and stratification. Am. J. Kidney Dis., 39 (2 Suppl 1), S1-266.

Reichenheim, M.E. (2004) Confidence intervals for the kappa statistic. Stata J., 4, 421-428.

Schieppati, A. \& Remuzzi, G. (2005) Chronic renal diseases as a public health problem: epidemiology, social, and economic implications. Kidney Int. Suppl., S7-S10.

Sim, J. \& Wright, C.C. (2005) The kappa statistic in reliability studies: use, interpretation, and sample size requirements. Phys. Ther, 85, 257-268.

Smith, E.R., Cai, M.M., McMahon, L.P., Wright, D.A. \& Holt, S.G. (2012) The value of simultaneous measurements of urinary albumin and total protein in proteinuric patients. Nephrol. Dial. Transplant., 27, 1534-1541.

Terawaki, H., Nakayama, M., Asahi, K., Kakamu, T., Hayakawa, T., Iseki, K., Kimura, K., Moriyama, T., Yamagata, K., Narita, I., Fujimoto, S., Tsuruya, K., Konta, T., Kondo, M., Kurahashi, I., et al. (2014) Comparison of predictive value for first cardiovascular event between Japanese GFR equation and coefficient-modified CKD-EPI equation. Clin. Exp. Nephrol., 19, 387-394

Viera, A.J. \& Garrett, J.M. (2005) Understanding interobserver agreement: the kappa statistic. Fam. Med., 37, 360-363.

World Health Organization (2011) Political Declaration of the High-level Meeting of the General Assembly on the Prevention and Control of Non-communicable Diseases. http://www.who.int/nmh/events/un_ncd_summit2011/political declaration_en.pdf [Accessed: August 10, 2015].

Yamamoto, K., Komatsu, Y., Yamamoto, H., Izumo, H., Sanoyama, K., Monden, M., Takeda, K., Nakahara, F. \& Yoshida, K. (2011) Establishment of a method to detect microalbuminuria by measuring the total urinary protein-to-creatinine ratio in diabetic patients. Tohoku J. Exp. Med., 225, 195-202.

Yamamoto, K., Yamamoto, H., Yoshida, K., Niwa, K., Nishi, Y., Mizuno, A., Kuwabara, M., Asano, T., Sakoda, K., Niinuma, H., Nakahara, F., Takeda, K., Shindoh, C. \& Komatsu, Y. (2014) The total urine protein-to-creatinine ratio can predict the presence of microalbuminuria. PLoS One, 9, e91067. 